\title{
Lipingshu capsule improves atherosclerosis associated with lipid regulation and inflammation inhibition in apolipoprotein E-deficient mice
}

Jiqu Xu' ${ }^{1,2,3}$, Congcong Ma ${ }^{1,2,3}$, Meng Chen ${ }^{1,2,3}$, Shuang Rong ${ }^{4}$ Hui Gao ${ }^{5}$, Zumeng Xia ${ }^{6 *}$ and Fenghong Huang ${ }^{1,2,3^{*}}$

\begin{abstract}
Background: Atherosclerosis (AS) is mainly responsible for cardiovascular diseases. The present study investigated whether Lipingshu capsule (LPS), whose ingredients are present in health food stores, has beneficial effect on AS.

Methods: C57BL/6 J mice were given a low fat rodent diet and assigned as control group (CON). ApoE ${ }^{-/-}$mice were placed on high fat rodent diet and randomly separated into high fat diet (HFD) group and HFD + LPS group whose animals were given $0.9 \mathrm{~g} / \mathrm{kg}$.BW LPS daily for 10 weeks. Atherosclerotic lesions in aorta and aortic root were evaluated. Serum lipids and multiple cytokine were measured.

Results: $\mathrm{ApoE}^{-/-}$mice fed with high fat diet had serious aortic lesions, whereas LPS markedly decreased plaque area of the total aorta and of the aortic root. LPS recovered the serum lipid profiles by substantially reducing TC, LDL-C, TG and Ox-LDL contents. Multi-cytokine analysis revealed greater serum levels of IL-1a, IL-1 $\beta, I L-6, I F N-\gamma$, GMCSF, RANTES and TNF-a induced by high fat diet slumped with LPS treatment.
\end{abstract}

Conclusion: LPS reduces atherosclerotic lesions and thus alleviates AS by lipid profile modulation and inflammation inhibition.

Keywords: Lipingshu, Capsule, Atherosclerosis, Lipid profile, Inflammation

\section{Background}

Cardiovascular diseases (CVD), representing 31\% of all global deaths, are the first cause of death worldwide according to updated statistics (source: http://www.who.int/ mediacentre/factsheets/fs317/en/ accessed Updated May 2017). Atherosclerosis (AS) is the lesion central to CVD including sudden cardiac death, myocardial infarction, unstable angina, peripheral thromboses and stroke. It has been identified as a complex disease process resulting from the interaction between lipid perturbations and inflammation [1].

\footnotetext{
* Correspondence: Zumeng.Xia@infinitus-int.com; fhhuang@foxmail.com ${ }^{6}$ Functional Oil Laboratory Associated by Oil Crops Research Institute, Chinese Academy of Agricultural Sciences and Infinite (China) Co., LTD., 66 Jianzhong Road, Guangzhou 510665, People's Republic of China

'Department of Nutriology, Oil Crops Research Institute, Chinese Academy of Agricultural Sciences, 2 Xudong Second Road, Wuhan 430062, People's Republic of China

Full list of author information is available at the end of the article
}

Dyslipidemia and inflammation are major mechanisms involved in the pathophysiology of atherosclerosis. Hyperlipidemia is characterized serologically by increased plasma triglyceride (TG), total cholesterol (TC) and low density lipoprotein cholesterol (LDL-C) levels [2]. Hypercholesterolemia is unique in being sufficient to result in atherosclerotic damage, even in the absence of other cardiovascular risk factors [3]. Elevated circulating LDL-C level is recognized as the major risk factor for AS, as supported by clinical evidence showing decreased atherosclerotic disease events when LDL-C was therapeutically lowered. The LDL oxidative modification (oxLDL) is crucial in atherogenesis [3-5] since oxLDL promotes AS progression directly by many mechanisms [3, 6-8] and served as a biomarker of CVD [9]. Evidence suggests that hypertriglyceridemia may also pose a significant risk for CVD [10, 11]. AS is likewise a chronic a chronic inflammatory process. Many risk factors such as

(C) The Author(s). 2018 Open Access This article is distributed under the terms of the Creative Commons Attribution 4.0 International License (http://creativecommons.org/licenses/by/4.0/), which permits unrestricted use, distribution, and 
dyslipidemia, hypertension and obesity trigger multiple inflammatory reactions, which lead to monocytes recruitment and foam cells formation in AS lesions [12]. The interactions between disordered lipid metabolism and inflammatory processes aggravate the development of AS [1].

Traditional Chinese Medicine (TCM) has been widely used for thousands of years in China, and now it is also deemed to treat various diseases or promote health. Some TCM formulas have been reported to possess antiatherosclerotic properties $[13,14]$. As a potential ingredient of functional food, there are direct evidences for the effects of milk-derived bioactive peptides against atherosclerosis by many mechanisms including anti-inflammatory and hypolipidemic activities [15-17]. Lipingshu capsule (LPS) is an innovative formula and its major ingredients include Gastrodia elata Bl.(Tianma), Eucommia ulmoides Oliver (Duzhong), Apocyniveneti Folium (Luobumaye) and milk-derived peptides, which all served as ingredients for health food in china (http://samr.cfda.gov.cn/WS01/ CL1160/76528.html). The most important active substances of LPS are gastrodin $(0.37 \% \mathrm{~W} / \mathrm{W})$, flavones $(2.2 \%$
$\mathrm{W} / \mathrm{W}$ ) and peptides and these ingredients may influence several risk factors for cardiovascular disease. For example, Gastrodia elata Bl. extract suppresses TNF- $\alpha$-induced vascular inflammatory process and matrix metalloproteinase activity in endothelial cells $[18,19]$. The extract of Eucommia ulmoides Oliver exhibits antihyperlipidemic properties by suppressing hepatic cholesterol and fatty acid biosynthesis [20] and iridoid and catechol derivatives from Eucommia ulmoides Oliver exert beneficial effects on inflammation regulation [21]. In addition, milk-derived bioactive peptides [22], the extracts of Eucommia ulmoides Oliver [23] and Apocyniveneti Folium [24] have been reported to possess anti-oxidant activities. Therefore, we determined whether this formula has a beneficial effect on atherosclerotic progression.

\section{Methods}

\section{Animals}

Twenty male $\mathrm{ApoE}^{-1-}$ mice on a $\mathrm{C} 57 \mathrm{BL} / 6 \mathrm{~J}$ background and 10 male wild-type (C57BL/6 J) mice were obtained from Vital River Laboratory Animal Technology Co., Ltd. (Beijing, China) at aged 7 weeks. The mice were
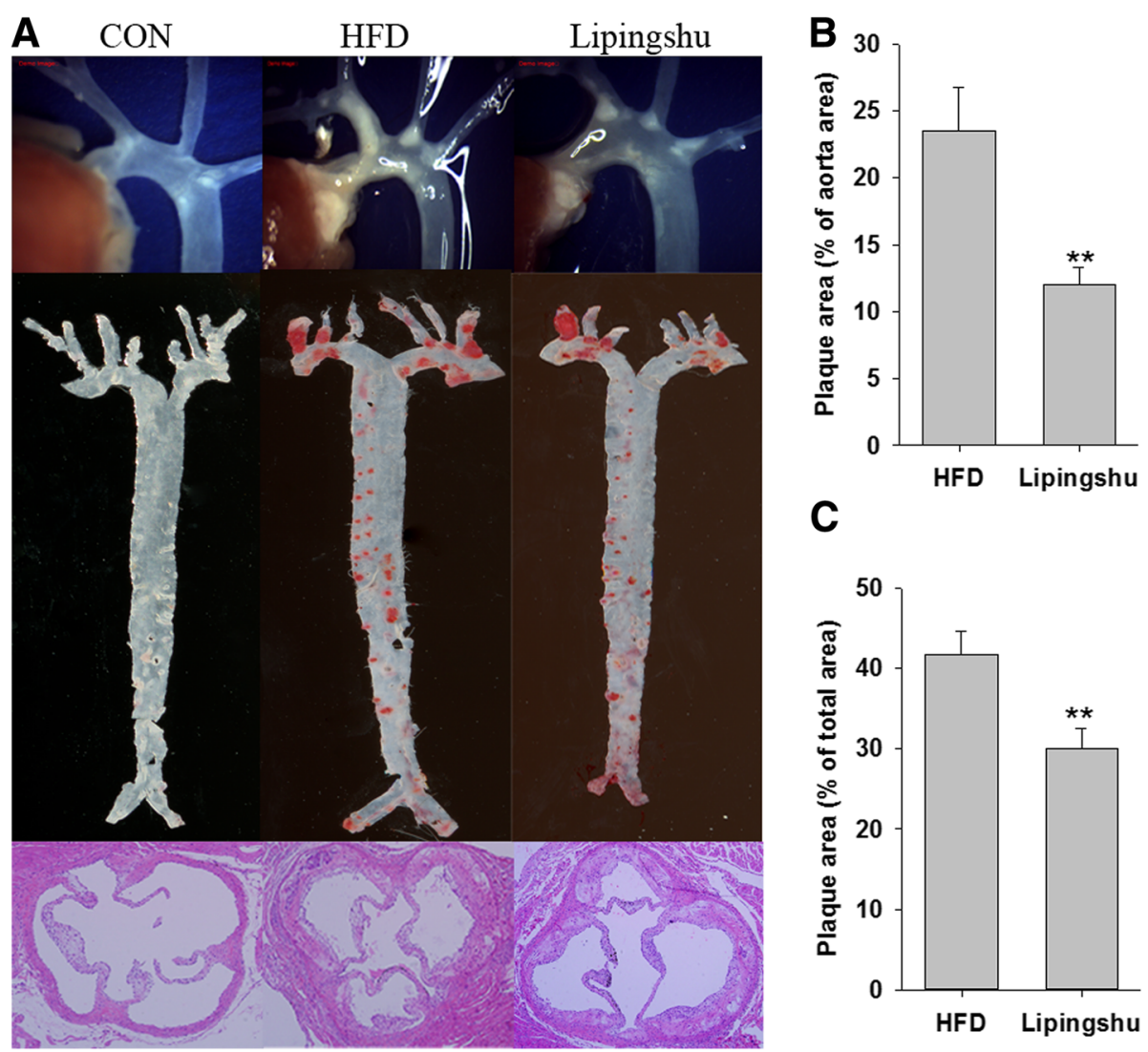

Fig. 1 Effects of Lipingshu capsule on atherosclerotic lesions. a. Representative photographs of aortic lesions (top), images of Oil Red O-stained (middle), and HE-stained aortic root lesions (bottom). b. Areas of the aortic lesion were expressed as the percentage of aorta areas. c. Areas of the aortic sinus lesion were expressed as the percentage of total areas. Bars represent mean \pm SEM ( $n=10$ animals/group). ${ }^{* *} p<0.01$ compared to the HFD group 
housed individually in climate-controlled rooms $\left(22 \pm 1^{\circ}\right.$ C) under diurnal conditions (light-dark: 08:00-20:00) with ad libitum access to tap water and standard laboratory rodent chow. After acclimatization for 7 days, the wild-type mice were fed a low fat diet (10 kcal\% fat, $0 \%$ cholesterol, Research Diets D12102C, USA) and assigned as control group (CON, $n=10)$. The $\mathrm{ApoE}^{-/-}$mice were fed high fat rodent diet (40 kcal\% fat, 1.25\% cholesterol, Research Diets D12108C, USA) and randomly assigned to two groups: high fat diet (HFD) group $(n=10)$ and Lipingshu capsule group $(n=10)$ whose mice received 0.9 g/kg.BW LPS (Infinite Co., LTD. China) orally by a gavage daily for 10 weeks. The animals were cared for in accordance with the Guiding Principles in the Care and Use of Animals.

\section{Lipids analysis}

For the measurement of blood lipids, the animals were fasted for $12 \mathrm{~h}$. Blood was collected from animals under deep anesthesia with isoflurane (1.5\%) via the retro-orbital venous plexus. Serum obtained by centrifuging at $1500 \mathrm{~g}$ $\left(10 \mathrm{~min}, 4{ }^{\circ} \mathrm{C}\right)$ was stored at $-80{ }^{\circ} \mathrm{C}$. The serum levels of TG, TC, LDL-C and high-density lipoprotein cholesterol (HDL-C) were measured by Hitachi 7020 full-automatic biochemical analyzer (Japan) with commercial kits (Wako, Japan). Serum oxLDL concentrations were analyzed with ELISA kit (Cloud-Clone, Houston, TX).

\section{Atherosclerosis}

The aorta was examined by stereo microscope, isolated and stripped of any external fatty deposits, then longitudinally opened and stained with Oil Red O (sigma, St. Louis, MO). The images are captured with a photo scanner (EPSON GT-X980). The total aortic surface area and Oil Red O-stained area were measured using Image-Pro Plus 6.0 (Media Cybernetics, Rockville, MD, USA).

After embedded with $4 \%$ paraformaldehyde, the aortic roots were consecutive sections (10 um) from aortic sinus to aortic arch. Five sections taken at 80 um intervals were examined under a light microscope (Olympus BX61) after staining with hematoxylin-eosine (H\&E). The lesion size was determined with Image-Pro Plus 6.0 (Media Cybernetics, Rockville, MD, USA).

\section{Multiple cytokine measurements}

The serum cytokines levels were measured with ProcartaPlex Mouse Cytokine \& Chemokine Convenience Panel 1A (36-plex, Thermo Fisher Scientific, Waltham, MA, USA) from eBioscience following the manufacturer's instructions.

\section{Statistical analyses}

Results are presented as mean \pm SEM. Statistical analysis was performed based on t-test and one-way ANOVA using SPSS 13.0 statistical software (SPSS Inc., Chicago, IL). The difference was considered significant when $p<0.05$.
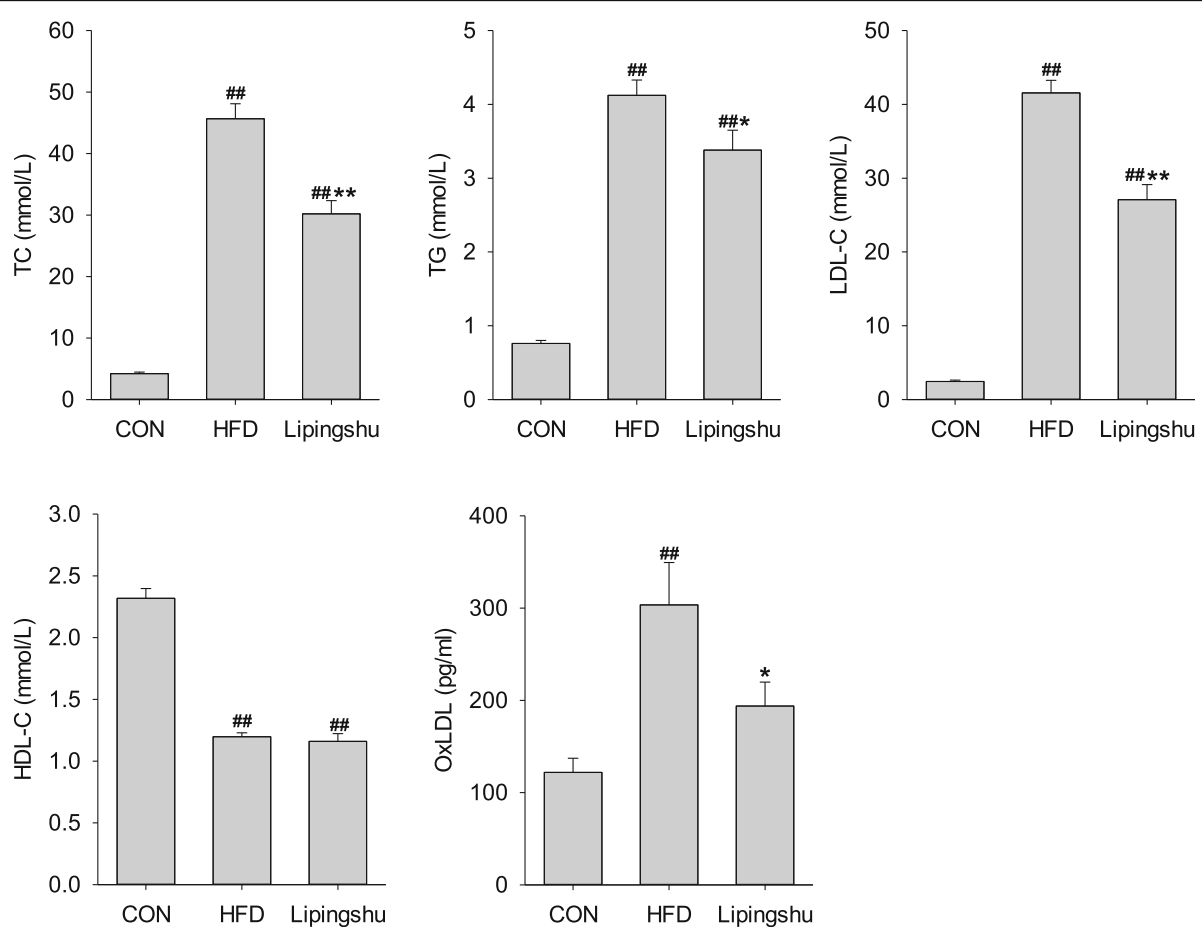

Fig. 2 Effects of Lipingshu capsule on the serum lipid profiles. Bars represent mean $\pm \operatorname{SEM}(n=10)$. \#\# $p<0.01$ compared to the CON group, * $p<0.05$ and ${ }^{* *} p<0.01$ compared to the HFD group 


\section{Results}

\section{Effects of LPS on atherosclerotic plaques}

As shown in Fig. 1a, there was no obvious atherogenesis observed by stereo microscope in CON animals. However, $\mathrm{ApoE}^{-/-}$mice fed a HFD had severe aortic lesions. Unanimously, Oil Red O-stained aortas as well as $\mathrm{H} \& \mathrm{E}$--stained aortic roots both showed pronounced aortic atherosclerotic plaque development in HFD group. The development of aortic lesions was regressed with LPS treatment. The quantitative analysis (Fig. 1b and c) revealed that the plaque areas of the total aorta and aortic root significantly declined in HFD + LPS group.

\section{Effects of LPS on serum lipid profile}

$\mathrm{ApoE}^{-/-}$mice receiving the HFD considerably heightened the serum levels of TG, TC and LDL-C but slashed the HDL-C level when compared with the control animals (Fig. 2). LPS supplement substantially reduced the contents of serum TG, TC and LDL-C but did not significantly affect HDL-C level. OxLDL levels were remarkably higher in HFD-fed $\mathrm{ApoE}^{-/-}$mice compared with those of control mice, but LPS reduced serum oxLDL concentration significantly.

\section{Effects of LPS cytokine and chemokine levels}

To ascertain the systemic effects of LPS on chronic inflammatory response, multi-cytokine analysis was performed to test the levels of serum inflammatory cytokines and chemokines. ApoE ${ }^{-/-}$mice receiving the HFD had remarkably greater contents of interleukin-1alpha (IL-1 $\alpha)$, IL-1 $\beta$, IL-6, interferon $\gamma$ (IFN- $\gamma)$, Granulocyte-Macrophage Colony Stimulating Factor (GMCSF), regulated on activation, normal $\mathrm{T}$ cell expressed and secreted (RANTES), and tumour necrosis factor alpha (TNF- $\alpha$ ) than control animals (Fig. 3), and these inflammatory cytokines declined remarkably when treated with LPS. The remaining chemokines and cytokines levels were unaffected by LPS (data not shown).
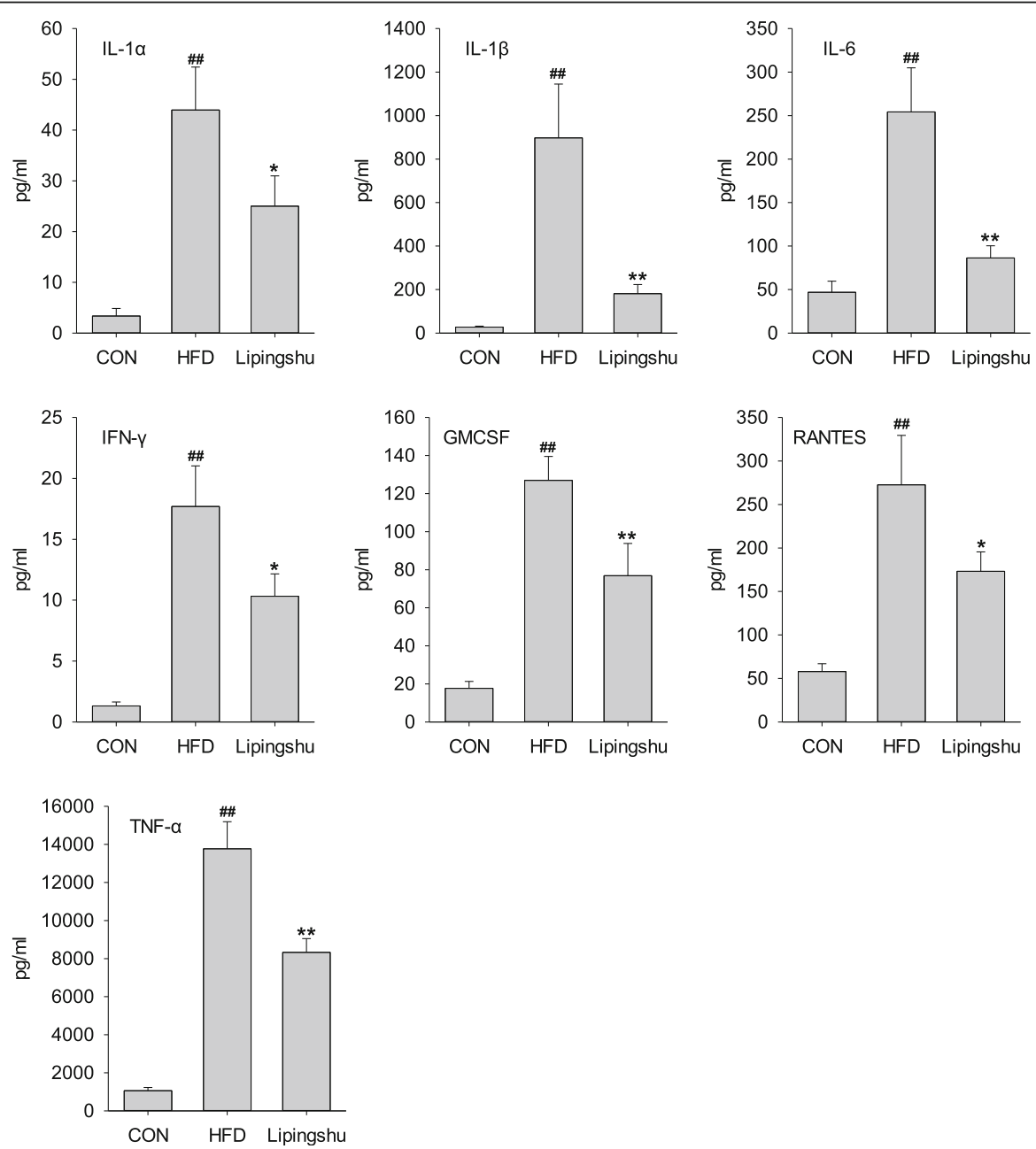

Fig. 3 Effects of Lipingshu capsule on serum cytokine and chemokine levels. Bars represent mean $\pm \operatorname{SEM}(n=10)$. \#\# $p<0.01$ compared to the CON group, ${ }^{*} p<0.05$ and ${ }^{* *} p<0.01$ compared to the HFD group 


\section{Discussion}

AS is the leading causes of CVD which cause most mortality and morbidity worldwide. Although some TCM herbal formula are valuable resources and therapeutic strategies for AS, the identification of their mechanism of action is a major challenge. The present study revealed that LPS, an innovative formula can be used in health food, possesses protective anti-atherosclerotic effects by improving serum lipids and inflammation.

HFD actually contributes to the development of AS in $\mathrm{ApoE}^{-/-}$mice. In the present study, HFD remarkably promote plaque formation in aortas as well as aortic sinus in atherosclerotic animals. LPS treatment abated atherosclerotic lesions, which was demonstrated by a dramatic diminution in atherosclerotic plaque formation. Hyperlipidemia is a major cause to accelerate AS progression of [25]. It has been widely demonstrated that raised TC and LDL-C contents are atherogenic [26, 27]. The oxidative modification of LDL-C and posterior foam cell formation from macrophage are the hallmarks and pivotal events in the pathogenesis of AS [28]. HDL is believed to possess cardioprotective properties [26, 29] by reversing cholesterol transport, protecting the vascular endothelium, impeding oxidative changes in LDL and exerting anti-inflammatory effects [30, 31]. Therefore, improvement of blood lipid profiles (including increasing HDL-C, reducing TC, LDL-C and TG, and demoting oxLDL generation) represents the most effective prevention strategy for AS. Some TCM herbals have been reported to prevent the lesion development of AS by regulating lipid profiles and blocking LDL oxidation $[13,32]$. In the present study, HFD increased serum TG, TC and LDL-C levels and decreased HDL-C level. LPS supplement markedly decreased TG, TC and LDL-C but did not engender HDL-C change in $\mathrm{ApoE}^{-/-}$mice, which leading to the decline in $\mathrm{TC} / \mathrm{HDL}-\mathrm{C}$ as well as LDL-C/HDL-C ratios [33] and thus underpinning the atherosclerotic protection. Extenuating raised ox-LDL levels in HFD-fed atherogenic animals suggested that inhibiting LDL oxidation is another therapeutic approach to AS for LPS.

Numerous basic research have established a prominent role for inflammation in the development of AS [12, 34] and now AS has been established as a chronic inflammatory disease [12]. In AS, the pernicious inflammatory response in atherosclerotic artery increases inflammatory cytokines and other acute-phase reactants contents in blood [34]. On the other hand, a specified group of circulating cytokines, such as TNF- $\alpha$, IL- $1 \alpha$, IL- $1 \beta$, IL-2 and IFN- $\gamma$, participates in initiating and sustaining full-blown AS [35]. So anti-inflammation is the new therapeutic option for treating AS [36]. In line with previous report [37], HFD induced AS progression was accompanied by marked increment of the pro-atherogenic cytokines, and LPS intervention repressed the excessive production of the inflammation cytokines (such as IL-1 $\alpha$, IL-1 $\beta$, IL-6, IFN- $\gamma$, GMCSF, RANTES and TNF- $\alpha$ ). Therefore, LPS may substantially prevent AS by its anti- inflammation activity.

\section{Conclusions}

In conclusion, LPS reduces atherosclerotic plaque burden and thus improves AS by lipid profiles regulation and anti-inflammation. Since all the ingredients of the formula are available in health food stores, LPS may act as a functional food to inhibit AS development and provide effective protection for CVD.

\section{Abbreviations \\ ANOVA: Analysis of variance; AS: Atherosclerosis; CVD: Cardiovascular diseases; GMCSF: Granulocyte-Macrophage Colony Stimulating Factor; HDL- C: high-density lipoprotein cholesterol; HFD: High fat diet; IFN- $\gamma$ : Interferon $\gamma$; IL: Interleukin; LDL-C: Low density lipoprotein cholesterol; oxLDL: LDL Oxidative modification; PS: Lipingshu capsule; RANTES: Regulated on activation, normal T cell expressed and secreted; TC: Total cholesterol; TCM: Traditional Chinese Medicine; TG: Triglyceride; TNF-a: Tumour necrosis factor alpha}

\section{Acknowledgments}

We would like to thank Xiaoming Zhou for his advice during histopathological examination.

\section{Funding}

This work was supported by Major Project of Technology Innovation Program of Hubei Province, China (2017ABA144) and Agricultural Science and Technology Innovation Project of Chinese Academy of Agricultural Sciences (CAAS-ASTIP-2013-OCRI).

\section{Availability of data and materials}

The datasets supporting the conclusions of this article are included within the article.

\section{Authors' contributions}

JX and FH designed the overall research experiments. CM, MC, HG, and SR performed the experiments and data analysis. ZX collected and analyzed the references. JX and FH wrote and edited the manuscript. All authors read and approved the final manuscript.

\section{Ethics approval}

The experiment was approved by the Oil Crops Research Institute Council on Animal Care Committee, Chinese Academy of Agricultural Sciences ([2015] IACUC Number: 0016)

Consent for publication

The authors declare that they agree to publish the paper.

\section{Competing interests}

The authors declare that they have no competing interests.

\section{Publisher's Note}

Springer Nature remains neutral with regard to jurisdictional claims in published maps and institutional affiliations.

\section{Author details}

${ }^{1}$ Department of Nutriology, Oil Crops Research Institute, Chinese Academy of Agricultural Sciences, 2 Xudong Second Road, Wuhan 430062, People's Republic of China. ${ }^{2}$ Hubei Key Laboratory of Lipid Chemistry and Nutrition, Oil Crops Research Institute, Chinese Academy of Agricultural Sciences, 2 Xudong Second Road, Wuhan 430062, People's Republic of China. ${ }^{3}$ Key Laboratory of Oilseeds processing, Ministry of Agriculture, 2 Xudong Second Road, Wuhan 430062, People's Republic of China. ${ }^{4}$ Department of Nutrition and Food Hygiene, School of Public Health, Medical College, Wuhan 
University of Science and Technology, No. 2, Huangjiahu Road, Wuhan 430065, China. ${ }^{5}$ Department of Nutrition and Food HygieneSchool of Public Health, Tongji Medical College, Huazhong University of Science and Technology, 13 Hangkong Road, Wuhan 430030, People's Republic of China. ${ }^{6}$ Functional Oil Laboratory Associated by Oil Crops Research Institute, Chinese Academy of Agricultural Sciences and Infinite (China) Co., LTD., 66 Jianzhong Road, Guangzhou 510665, People's Republic of China.

Received: 24 April 2018 Accepted: 10 July 2018

Published online: 31 July 2018

\section{References}

1. van Diepen JA, Berbee JF, Havekes LM, Rensen PC. Interactions between inflammation and lipid metabolism: relevance for efficacy of antiinflammatory drugs in the treatment of atherosclerosis. Atherosclerosis. 2013:228:306-15.

2. Tonstad S, Despres JP. Treatment of lipid disorders in obesity. Expert Rev Cardiovasc Ther. 2011:9:1069-80.

3. Glass CK, Witztum JL. Atherosclerosis. the road ahead. Cell. 2001;104:503-16.

4. Gosmanova EO, Le NA. Cardiovascular complications in CKD patients: role of oxidative stress. Cardiol Res Pract. 2011;2011:156326.

5. Jones NL, Reagan JW, Willingham MC. The pathogenesis of foam cell formation: modified LDL stimulates uptake of co-incubated LDL via macropinocytosis. Arterioscler Thromb Vasc Biol. 2000;20:773-81.

6. Maiolino G, Rossitto G, Caielli P, Bisogni V, Rossi GP, Calo LA. The role of oxidized low-density lipoproteins in atherosclerosis: the myths and the facts. Mediat Inflamm. 2013;2013:714653.

7. Liu J, Ren Y, Kang L, Zhang L. Oxidized low-density lipoprotein increases the proliferation and migration of human coronary artery smooth muscle cells through the upregulation of osteopontin. Int J Mol Med. 2014;33:1341-7.

8. Steinberg D, Parthasarathy S, Carew TE, Khoo JC, Witztum JL. Beyond cholesterol. Modifications of low-density lipoprotein that increase its atherogenicity. N Engl J Med. 1989;320:915-24

9. Trpkovic A, Resanovic I, Stanimirovic J, Radak D, Mousa SA, Cenic-Milosevic D, Jevremovic D, Isenovic ER. Oxidized low-density lipoprotein as a biomarker of cardiovascular diseases. Crit Rev Clin Lab Sci. 2015:52:70-85.

10. Matsumoto S, Gotoh N, Hishinuma S, Abe Y, Shimizu Y, Katano Y, Ishihata A. The role of hypertriglyceridemia in the development of atherosclerosis and endothelial dysfunction. Nutrients. 2014;6:1236-50.

11. Le NA, Walter MF. The role of hypertriglyceridemia in atherosclerosis. Curr Atheroscler Rep. 2007;9:110-5.

12. Libby P, Ridker PM, Maseri A. Inflammation and atherosclerosis. Circulation. 2002;105:1135-43

13. Cheng $L$, Pan GF, Zhang XD, Wang JL, Wang WD, Zhang JY, Wang $H$, Liang RX, Sun XB. Yindanxinnaotong, a Chinese compound medicine, synergistically attenuates atherosclerosis progress. Sci Rep. 2015;5:12333.

14. Zheng J, Liu B, Lun Q, Gu X, Pan B, Zhao Y, Xiao W, Li J, Tu P. Longxuetongluo capsule inhibits atherosclerosis progression in high-fat diet-induced ApoE-/- mice by improving endothelial dysfunction. Atherosclerosis. 2016;255:156-63.

15. Aihara K, Ishii H, Yoshida M. Casein-derived tripeptide, Val-pro-pro (VPP), modulates monocyte adhesion to vascular endothelium. J Atheroscler Thromb. 2009:16:594-603.

16. Nakamura T, Hirota T, Mizushima K, Ohki K, Naito Y, Yamamoto N, Yoshikawa T. Milk-derived peptides, Val-pro-pro and Ile-pro-pro, attenuate atherosclerosis development in apolipoprotein e-deficient mice: a preliminary study. J Med Food. 2013;16:396-403.

17. Marcone S, Belton O, Fitzgerald DJ. Milk-derived bioactive peptides and their health promoting effects: a potential role in atherosclerosis. $\mathrm{Br} \mathrm{J}$ Clin Pharmacol. 2017:83:152-62.

18. Hwang SM, Lee YJ, Kang DG, Lee HS. Anti-inflammatory effect of Gastrodia elata rhizome in human umbilical vein endothelial cells. Am J Chin Med. 2009;37:395-406.

19. Lee YJ, Hwang SM, Kang DG, Kim JS, Lee HS. Effect of Gastrodia elata on tumor necrosis factor-alpha-induced matrix metalloproteinase activity in endothelial cells. J Nat Med. 2009;63:463-7.

20. Choi MS, Jung UJ, Kim HJ, Do GM, Jeon SM, Kim MJ, Lee MK. Du-zhong (Eucommia ulmoides Oliver) leaf extract mediates hypolipidemic action in hamsters fed a high-fat diet. Am J Chin Med. 2008;36:81-93.
21. Luo D, Or TC, Yang CL, Lau AS. Anti-inflammatory activity of iridoid and catechol derivatives from Eucommia ulmoides Oliver. ACS Chem Neurosci. 2014;5:855-66

22. Mohanty DP, Mohapatra S, Misra S, Sahu PS. Milk derived bioactive peptides and their impact on human health - a review. Saudi J Biol Sci. 2016;23:577-83.

23. He X, Wang J, Li M, Hao D, Yang Y, Zhang C, He R, Tao R. Eucommia ulmoides Oliv.: ethnopharmacology, phytochemistry and pharmacology of an important traditional Chinese medicine. J Ethnopharmacol. 2014;151:78-92.

24. Xie W, Zhang X, Wang T, Hu J. botany, traditional uses, phytochemistry and pharmacology of Apocynum venetum L. (Luobuma): a review. J Ethnopharmacol. 2012;141:1-8.

25. McKenney JM. Pharmacotherapy of dyslipidemia. Cardiovasc Drugs Ther. 2001:15:413-22.

26. Castelli WP, Garrison RJ, Wilson PW, Abbott RD, Kalousdian S, Kannel WB. Incidence of coronary heart disease and lipoprotein cholesterol levels. The Framingham Study. JAMA. 1986;256:2835-8.

27. Kannel WB, Neaton JD, Wentworth D, Thomas HE, Stamler J, Hulley SB, Kjelsberg MO. Overall and coronary heart disease mortality rates in relation to major risk factors in 325, 348 men screened for the MRFIT. Multiple Risk Factor Intervention Trial. Am Heart J. 1986;112:825-36.

28. Linton MF, Fazio S. Macrophages, inflammation, and atherosclerosis. Int J Obes Relat Metab Disord. 2003;27(Suppl 3):S35-40.

29. Gordon T, Castelli WP, Hjortland MC, Kannel WB, Dawber TR. High density lipoprotein as a protective factor against coronary heart disease. The Framingham Study. Am J Med. 1977;62:707-14.

30. Annema W, von Eckardstein A. High-density lipoproteins. Multifunctional but vulnerable protections from atherosclerosis. Circ J. 2013;77:2432-48.

31. Hu J, Xi D, Zhao J, Luo T, Liu J, Lu H, Li M, Xiong H, Guo Z. High-density lipoprotein and inflammation and its significance to atherosclerosis. Am J Med Sci. 2016;352:408-15.

32. Liu G, Wang B, Zhang J, Jiang H, Liu F. Total panax notoginsenosides prevent atherosclerosis in apolipoprotein E-knockout mice: role of downregulation of CD40 and MMP-9 expression. J Ethnopharmacol. 2009; 126:350-4.

33. Lemieux I, Lamarche B, Couillard C, Pascot A, Cantin B, Bergeron J, Dagenais GR, Despres JP. Total cholesterol/HDL cholesterol ratio vs LDL cholesterol/ $\mathrm{HDL}$ cholesterol ratio as indices of ischemic heart disease risk in men: the Quebec cardiovascular study. Arch Intern Med. 2001;161:2685-92.

34. Hansson GK. Inflammation, atherosclerosis, and coronary artery disease. N Engl J Med. 2005;352:1685-95.

35. Kleemann R, Zadelaar S, Kooistra T. Cytokines and atherosclerosis: a comprehensive review of studies in mice. Cardiovasc Res. 2008;79:360-76.

36. Bertrand MJ, Tardif JC. Inflammation and beyond: new directions and emerging drugs for treating atherosclerosis. Expert Opin Emerg Drugs. 2017 22:1-26.

37. Naura AS, Hans CP, Zerfaoui M, Errami Y, Ju J, Kim H, Matrougui K, Kim JG, Boulares $\mathrm{AH}$. High-fat diet induces lung remodeling in ApoE-deficient mice: an association with an increase in circulatory and lung inflammatory factors. Lab Investig. 2009;89:1243-51.
Ready to submit your research? Choose BMC and benefit from:
- fast, convenient online submission
- thorough peer review by experienced researchers in your field
- rapid publication on acceptance
- support for research data, including large and complex data types
- gold Open Access which fosters wider collaboration and increased citations
- maximum visibility for your research: over $100 \mathrm{M}$ website views per year
At $\mathrm{BMC}$, research is always in progress. 